\title{
Comparison of the gas-liquid dual support fixation and Heitzman fixation techniques for preparing lung specimens
}

\author{
DONGSHENG YU ${ }^{1}$, WEILI QU ${ }^{2}$, HAIPENG XIA ${ }^{3}$, XIAOFENG LI $^{1}$, ZHENFENG LUAN $^{1}$, \\ RENJIE YAN ${ }^{4}, \mathrm{XIAODONG} \mathrm{LU}^{5}$ and PENG ZHAO \\ ${ }^{1}$ Department of Intervention, Qilu Hospital of Shandong University, Qingdao, Shandong 266035; ${ }^{2}$ Department of \\ Cardiac Surgery, The Affiliated Hospital of Qingdao University, Qingdao, Shandong 266003; \\ ${ }^{3}$ Department of Orthopedics, Qilu Hospital of Shandong University, Qingdao, Shandong 266035; ${ }^{4}$ Department of \\ Intervention, Beijing Tumor Hospital, Beijing 100036; Departments of ${ }^{5}$ Radiology and ${ }^{6}$ Pathology, \\ The Affiliated Hospital of Qingdao University, Qingdao, Shandong 266003, P.R. China
}

Received December 21, 2015; Accepted March 10, 2017

DOI: $10.3892 /$ etm.2017.4563

\begin{abstract}
The aim of the present study was to compare the gas-liquid dual support fixation and Heitzman fixation techniques for the preparation of lung specimens. A total of 40 fresh lung samples were surgically collected from 40 male patients with lung cancer by biopsy. Patients were recruited from the Affiliated Hospital of Qingdao University Medical College (Qingdao, China) between July 2007 and June 2014. Samples were prepared using either the gas-liquid dual support fixation method (group A; $n=26$ ) or the Heitzman fixation method (group B; n=14). High-resolution computed tomography (HRCT) scanning was performed prior to surgery and corresponding postoperative HRCT scanning was conducted for the lung specimens; the gross transverse specimen section, cord photography images and histological sections were evaluated. Morphological observations of lung specimens indicated that there were 22 cases in group A with grade I (84.6\%) and 4 cases with grade II (15.4\%), whereas, in group B, there were 5 cases with grade II (35.7\%) and 9 cases with grade III (64.3\%). Statistical analysis demonstrated that the grades of specimens between the two groups were significantly different $(\mathrm{P}<0.01)$. Results from imaging and histological studies found that the quality of lung specimens was superior in group A, compared with group B. In conclusion, the present study demonstrated that, compared with the Heitzman fixation method, gas-liquid dual support fixation may be a superior technique for the
\end{abstract}

Correspondence to: Professor Dongsheng Yu, Department of Intervention, Qilu Hospital of Shandong University, 758 Hefei Road, Qingdao, Shandong 266035, P.R. China

E-mail: shengyudongcn@126.com

Key words: gas-liquid dual support fixation, heitzman fixation, lung, specimen preparation of lung specimens. This finding may facilitate the improvement of lung HRCT and pathological studies.

\section{Introduction}

Lung cancer is a leading cause of cancer-associated mortality in adults globally and its incidence continues to rise (1). Preparation of lung specimens, which is considered the most important pre-analytical step, is key for pathologists to correctly diagnose and treat lung cancer, as well as provide an accurate prognosis for patients (2). Therefore, numerous imaging studies of pathological specimens have been conducted (1-4). However, compared with analytics, where advances over the last decade have led to a novel portfolio of test methods, developments in pre-analytics have been minimal (3). Since 1985, high-resolution computed tomography (HRCT) has been widely used in clinical practice and researchers have performed control studies of lung HRCT manifestations and pathological specimens (4-11), making HRCT an important tool for individual patient management.

Bronchoscopies with endobronchial ultrasonography and electromagnetic navigation are examples of novel technologies that have improved the specificity and sensitivity of HRCT in diagnosing and staging lung cancer (12). However, despite these advances in diagnostic tools, pathology remains the golden standard for diagnosing cancer. The Heitzman method, which is widely used for lung specimen fixation, requires preservation of the pleural integrity of lung specimens (13). Furthermore, owing to certain other limitations of this technique, the range of clinical applications on the source of specimens available is limited. The current study focuses on a novel method of lung specimen preparation, the gas-liquid dual support fixation method, which provides a constant distending pressure on the lung specimens to be fixed. This significantly lowers lung expansion, alveolar septal tissue volume, surface areas and diffusing capacity of the tissue-plasma barrier compared with other conventional methods (14). The current study aimed to obtain fixed specimens that are as close to metabolically active human lung tissue, which would be more conducive to imaging and pathological studies. 


\section{Materials and methods}

Specimens. Fresh lung lobe tissues were obtained via lobectomy from 40 male patients with lung cancer by biopsy. Patients were recruited from the Affiliated Hospital of Qingdao University (Qingdao, China) between July 2007 and June 2014. Computed tomography (CT) scanning was performed prior to surgery with continuous scanning being conducted at $1-\mathrm{cm}$ thickness and $10-\mathrm{mm}$ intervals to determine the distribution of bronchial arteries and veins. The lobes were resected and HRCT scanning was subsequently performed using the PQ 2000 rapid spiral CT scanner (Picker International, Highland Heights, OH, USA). Scan parameters were: Tube voltage, $140 \mathrm{kV}$; tube current, 130-170 mA; and scan time, 2 sec. Continuous HRCT scanning was conducted at $1.5-\mathrm{mm}$ thickness and $10-\mathrm{mm}$ intervals. The bone remodeling algorithm (15) was also used. Following scanning, five typical images were selected to identify layers marked by the bronchus, artery and vein. A specific point at each layer was selected to measure $\mathrm{CT}$ values. Lobes were then divided into two groups, A and B, by stratified randomization, based on the patient's age, lesion size, location, resection site and extent of pleura damages. A total of 26 specimens were categorized into group A and 14 specimens were categorized into group B. General characteristics pertaining to the distribution of these two groups are presented in Table I. The present study was conducted with approval from the Ethics Committee of Shandong University (Qingdao, China) and conducted in accordance with the declaration of Helsinki. Written informed consent was obtained from all participants.

Gas-liquid dual support fixation method for group A. The stationary liquid contained polyethylene glycol 400 (Thermo Fisher Scientific, Inc., Waltham, MA, USA), 95\% ethanol, 40\% formalin and purified water at a 10:5:2:3 ratio. To inject the stationary liquid, a catheter, connected to an enema infuser (CAC-2000C; Corey Medical, Zhongzhou, China) through a three- or four-way pipe following ligation, was inserted in the bronchial orifice of lung lobe, with the help of the enema infuser. Stationary liquid and gas were slowly injected into the lung through the bronchus (upper lobe, $50-75 \mathrm{ml}$; lower and median-lower lobe, $70-100 \mathrm{ml}$ ), with the infusion pressure maintained at 3.0 KPa. When the specimen expanded fully, it was floated in a plastic bucket containing the same stationary fluid, with a wet gauze covering the specimen surface. Gas at a pressure of $1.5 \mathrm{KPa}$ was injected into the specimen allowing it to maintain its shape. Samples were subsequently re-injected with same volume of stationary fluid $1 \mathrm{~h}$ later and maintained for $36 \mathrm{~h}$. Following fixation, the lung specimens were taken out and suspended for the 24-h drying step and gas was re-injected at a pressure of $2.0-2.5 \mathrm{KPa}$ to maintain the shape of the specimens.

Heitzman method for group B. Lung specimens were prepared as previously described (16). A catheter was inserted into the lung lobes through bronchial orifices to slowly inject stationary liquid, comprised of polyethylene glycol 400 (Thermo Fisher Scientific, Inc.), 95\% ethanol, $40 \%$ formalin and purified water at a 10:5:2:3 ratio at a low-pressure. This was performed until pleural surface dilation was achieved. The lung lobe was
Table I. Characteristics of the two groups.

\begin{tabular}{lcc}
\hline & A group $(\mathrm{n}=26)$ & B group $(\mathrm{n}=14)$ \\
\hline Age (years) & 73 & 70 \\
Max & 31 & 34 \\
Min & 58 & 57 \\
Mean & 6.07 & 8.06 \\
SD & & \\
Sex (cases) & 23 & 12 \\
Male & 3 & 2 \\
Female & & 4 \\
Resection (cases) & 11 & 4 \\
Right upper lobe & 3 & \\
Right median and & & 4 \\
lower lobe & 9 & 2 \\
Site & 3 & \\
Left upper lobe & & \\
Left lower lobe &
\end{tabular}

Lung samples in the A group underwent the gas-liquid dual support fixation method, whereas the Heitzman method was used for group B.

then fixed in the same liquid at $25^{\circ} \mathrm{C}$ for $24 \mathrm{~h}$ for specimen preparation.

Hematoxylin-eosin staining protocol. Lung specimens were stained by hematoxylin-eosin staining. Rehydrated lung sections ( $2 \mathrm{~mm}$ thick) were stained with hematoxylin solution for 20-40 $\mathrm{min}$ at room temperature. Sections were washed in tap water for 1-5 min, then washed in $70 \%$ ethanol containing $1 \% \mathrm{HCl}$ to remove excess dye, then washed for a further 1-5 min in tap water. Sections were stained with eosin solution for $10 \mathrm{~min}$, then washed in tap water for 1-5 min. Sections were dehydrated, mounted and examined under a light microscope.

Evaluation of specimens. Inflated, fixed lung specimens from groups A and B were assessed by two experienced physicians performing gross examinations (Fig. 1), cross-sectional observations of fixed lung specimens from groups $\mathrm{A}$ and HRCT findings (Fig. 2) and histopathological section observations (Figs. 3 and 4) by light microscopy and soft ray radiography (Fig. 5). Specimens were classified into grades I, II and III, according to previously published criteria (16).

Grade I: Size, shape, and elasticity of the lung specimen were close to that of the live organ, with a smooth pleural surface. The lobule was clearly visible, and the gross cross-sectional specimen clearly exhibited an interlobular septum, pulmonary micro-arteries and veins, as well as the bronchus and other structures. The normal histological appearance and pathological changes, including changes in respiratory bronchioles, pulmonary micro-arteries and veins, and alveoli, were clearly visible under a microscope.

Grade II: Specimen exhibited a slight deformation, with a slightly wrinkled pleural surface, although the pulmonary lobule remained visible on the pleural surface. Interlobular septum as well as other structures were visible on the cross 

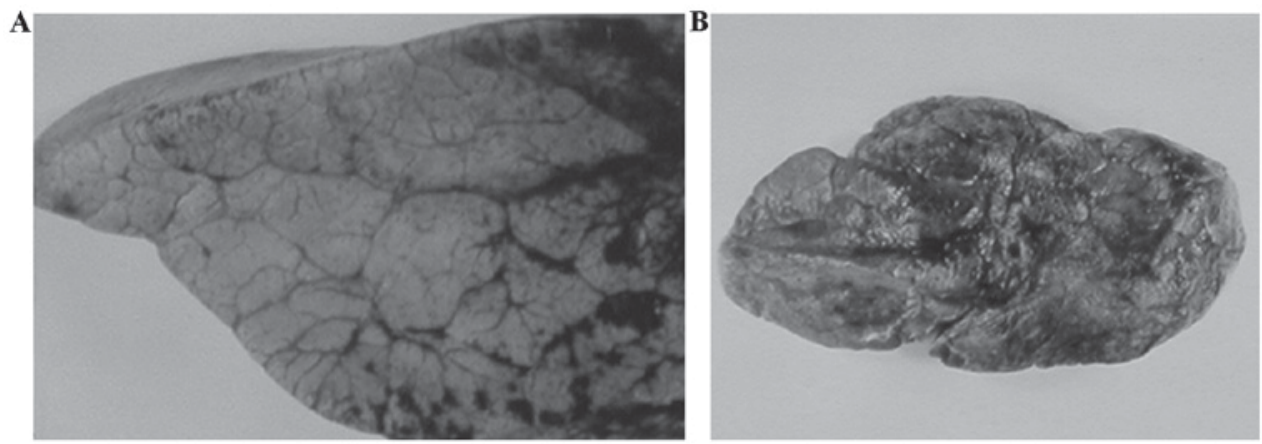

Figure 1. (A) Local gross specimen image of the upper lobe of the left lung in a 58-year-old male, prepared using the gas-liquid dual support fixation method. (B) Gross specimen image of the upper lobe of the left lung in a 60-year-old man, prepared using the Heitzman method.
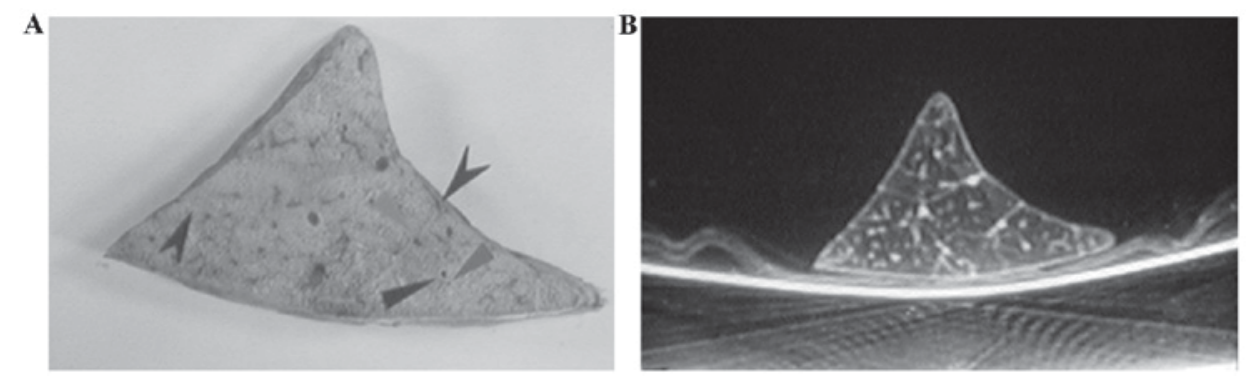

Figure 2. (A) Cross-sectional gross specimen image prepared by the gas-liquid dual support fixation method. The grey triangular arrow indicates the interlobular artery, the black triangular arrow indicates the lobular bronchus, the black double arrow on the right indicates the lobular vein and the black double arrow on the left indicates the interlobular septum. (B) Corresponding high-resolution computed tomography image of (A).
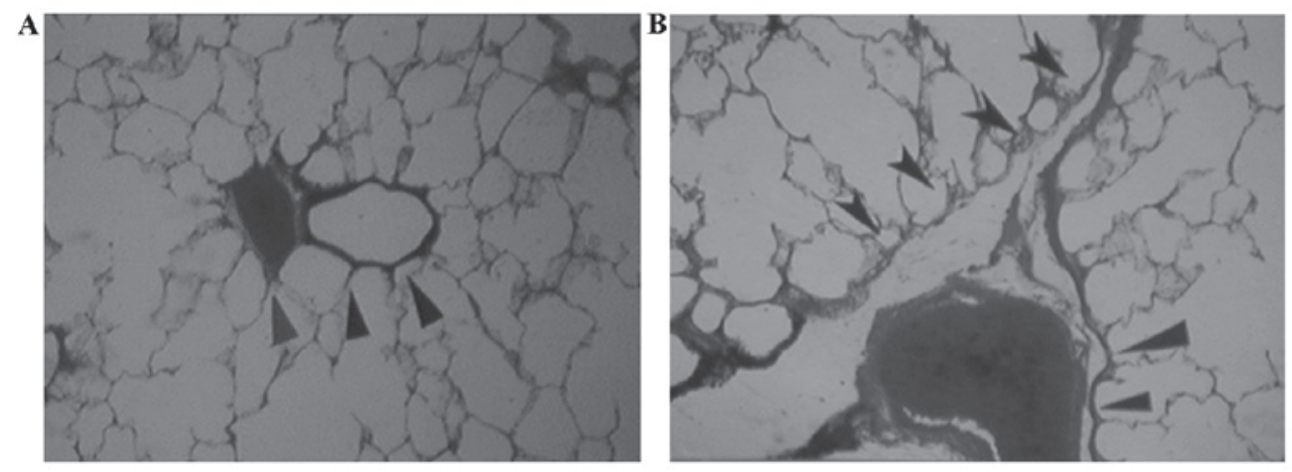

Figure 3. (A) Corresponding tissue section to Fig. 2A. Black arrow indicates the interlobular artery and black arrow indicates the lobular bronchus (hematoxylin-eosin staining; magnification, x40). (B) Corresponding tissue-slice image to Fig. 2A. Black triangular arrow indicates the lobular vein and black double arrow indicates the interlobular septum (hematoxylin-eosin staining; magnification, x100).
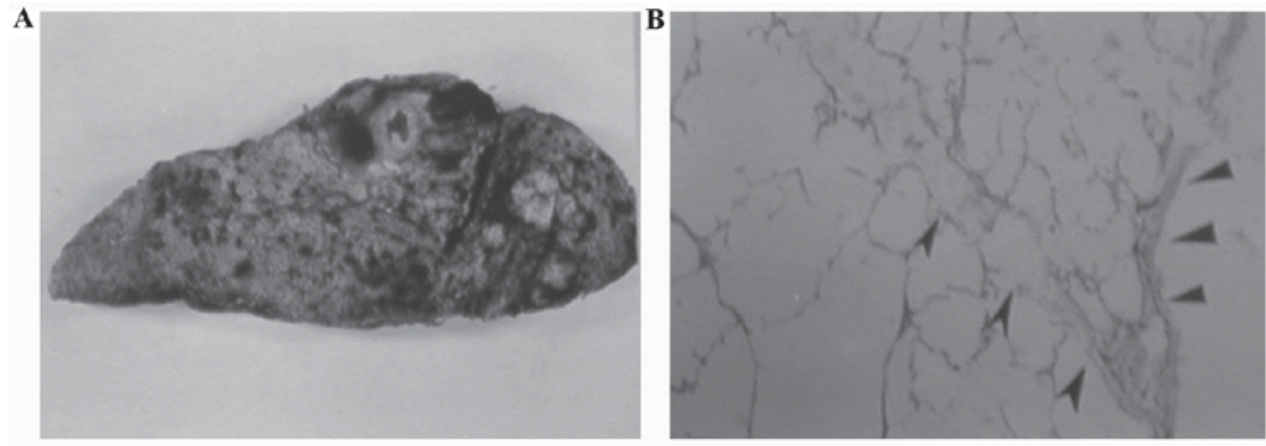

Figure 4. (A) Cross-sectional gross specimen image prepared using the Heitzman method. Lung slice is 1-cm thick. (B) Corresponding tissue section to Fig. 4. (A) of the Heitzman method (hematoxylin-eosin staining; magnification, $\mathrm{x} 40$ ). Black arrow indicates the pleura and black double arrow indicates the interlobular septum. Under the microscope, certain areas exhibited the folded alveolar intervals and atelectasis, whereas some regions exhibited the alveolar expansion and interval break. 


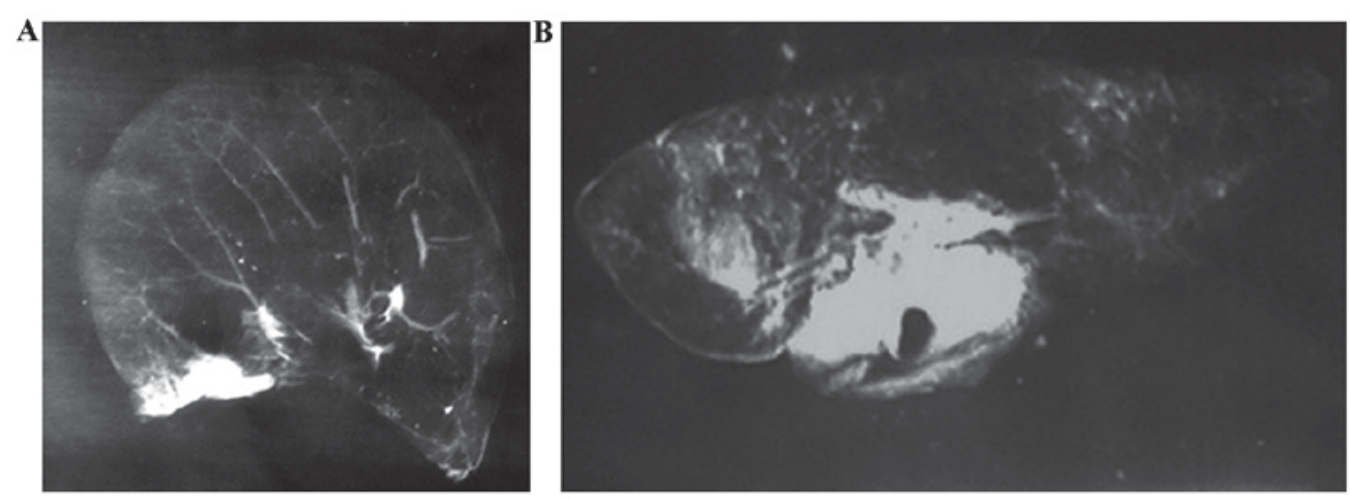

Figure 5. Soft-ray photographic images. (A) Lung section specimen (1 cm thick) from a patient in group A. (B) Lung section specimen (1 cm thick) from a patient in group B. Magnification, x1.4.

section of the gross specimen. Respiratory bronchioles and other histological and pathological features (such as alveolar ducts and alveolar structures) were visible under the microscope; and

Grade III: Specimen was deformed, with a shrunken pleural surface and most lobules on the pleural surface were not clearly visible. Cross section of the gross specimen revealed that partial areas of lung specimen were extruded and deformed and did not exhibit the fine anatomic structure of the lung. Under a microscope, the alveolar septa in the partial region appeared folded, with both the atelectasis and alveolus expanded in the partial region, and the interlobular septum appeared broken.

Statistical analysis. The Rank sum test was used to determine statistically significant differences between groups, according to the aforementioned grades. SPSS 19.0 software (SPSS, Inc., Chicago, IL, USA) was used for statistical analysis. $\mathrm{P}<0.01$ was considered to determine a statistically significant difference.

\section{Results}

Morphology. Quality evaluation results are presented in Table II. Significant differences were observed between groups $\mathrm{A}$ and $\mathrm{B}(\mathrm{P}<0.01)$ : in group $\mathrm{A}, 84.6$ and $15.4 \%$ of specimens were grade I and II, respectively, whereas, in group B, 0.0, 35.7, and $64.3 \%$ of specimens were grade I, II and III, respectively. These results indicate that preparation methods are influential on the quality of inflated fixed lung specimens. Table II suggests that the gas-liquid dual support method is superior to the Heitzman method in preparing inflated fixed lung specimens.

Imaging and histology. Fig. 2A shows a gross specimen prepared using the gas-liquid dual support fixation method. Fig. 2B shows the same specimen viewed using HRCT. Fig. 3 (H\&E histological staining, x40 magnification) presents two images of the same section of inflated fixed lung specimen prepared using the gas-liquid dual support fixation method. Fig. 3A presents interlobular arteries and bronchioles located in the center of the lobule and Fig. 3B presents the interlobular vein within the interlobular septum. The HRCT image exhibited a normal interlobular septum and interlobular arteries under the pleura and interlobular arteries located in the center of lobule, however lobular bronchioles could not be observed (Fig. 2A). When the interlobular septum was visible, interlobular arteries could be observed in the pulmonary lobule; however, the interlobular septum could not be observed in the middle of lung and its location could only be determined according to the central artery of interlobular septum (Fig. 2A). At the pulmonary lobule level, the pulmonary vein and artery had similar diameter $(0.1-0.2 \mathrm{~mm})$; the pulmonary vein traveled within the interlobular septum, making it difficult to determine its location (Fig. 3B). In group A, when the postoperative inflated fixed lung specimen was not completely dry, the interlobular septum in the central part of that lung could be clearly observed, possibly due to edema fluid causing thickening of the interlobular septum. When this specimen was completely dry, the interlobular septum was not clearly observed, although the bronchopulmonary arterial shadow that accompanies the interlobular septum was clearly observed (Fig. 1A). In group B, lung lobe specimens exhibited lobular structures (Fig. 1B); however, the quality was worse compared with specimens in group A, which showed decreased deformation (Fig. 4). In lung specimens in group B, a flocculent cloud high density shadow was often observed in local areas, due to the non-completely dried stationary fluid; additionally, lung shrinkage and deformation was observed, causing the basic lung structure to be non-displayable (Fig. 4). In the cross-section and incisal surface of the inflated fixed lung specimen, the bronchus and pulmonary arteries were clearly observed. The pulmonary vein entered the interlobular septum; however, due to the absence of the fillers, the deformation of pulmonary veins was irregular. Many small holes were visible on the cross-sectional surface, representing alveolar ducts and alveolar structures, and the interlobular septum expanded from the pleura to the lungs vertically or at an angle (Fig. 2A).

In the soft-ray photographic images (Fig. 5), 1-cm-thick lung section specimens of group A clearly exhibited progressive branching of naturally distributed pulmonary arteries, which were $\mathrm{V}$-shaped, reached 1-2 $\mathrm{cm}$ under the pleura and divided into a large capillary network. Meanwhile, in the partial region, the pulmonary vein was detected deep in the interlobular septum and the inflated bronchus exhibited low density, which impaired observation (Fig. 5A). In group B, the 1-cm-thick lung section specimens were contracted; thus, 
Table II. Quality evaluation of the two groups.

\begin{tabular}{lcll}
\hline Group, N $(\%)$ & Grade I & Grade II & Grade III \\
\hline A $(n=26)$ & $22(84.6)$ & $4(15.4)$ & $0(0.0)$ \\
B $(n=14)$ & $0(0.0)$ & $5(35.7)$ & $9(64.3)$ \\
\hline
\end{tabular}

Lung samples in the A group underwent the gas-liquid dual support fixation method, whereas the Heitzman method was used for group B.

pulmonary arteries were distributed unnaturally, appearing zigzag and earthworm-like, and the structure of pulmonary lobule was deformed (Fig. 5B).

\section{Discussion}

The results of the current study demonstrated that the quality of the lung specimen prepared by the gas-liquid dual support fixation method was better than that of specimens prepared by the Heitzman fixation method. Following gas-liquid dual support fixation, most specimens were grade I and no specimen was grade III; however, following Heitzman fixation, no specimen was grade I and all specimens were either grade II or grade III.

There are a number of factors affecting the preparation of inflated fixed lung specimens. Most lung lobes obtained by lobectomy are abandoned a few weeks after pathological examination and are thus rarely preserved. These lung lobe specimens are important materials for studies on lung anatomy and pathology, and are used to obtain lung HRCT images. However, due to pleural defects caused by the lobectomy, lung lobes can easily shrink and deform, thus limiting the application of various inflated lung specimen fixation techniques towards these materials (17-27). Many researchers have suggested improved methods of fixation: Heitzman (16) proposed the meticulous repair of small damaged pleural surfaces; Webb (1) proposed that lobe specimens should be floated on the surface of a stationary fluid; and proposed that in addition to the repair of the damaged pleura, the amount and pressure of the infused stationary fluid should be strictly controlled. However, the resulting inflated lung specimens were unsatisfactory after these fixation techniques. In the current study, by analyzing inflated lung specimens prepared by various techniques, it was determined that the Heitzman method, currently used by many scholars, exhibited notable disadvantages. As the Heitzman method involves the infusion of stationary mixture into the lung through the bronchus, it destroys the normal physiological state of lung tissues. Under the normal physiological state, the density of lung tissue is similar to that of air, while the density of lung tissue and blood is 1.055 , similar to that of water (17). Although the stationary mixture fixed cellular structures and supported the bronchial stent structures, during the air-drying process, the substitution of the supporting liquid by gas causes an abrupt conversion, leading to slight atrophy and deformation of inflated fixed lung specimens (17). Furthermore, the Heitzman method (18) involves filling the bronchial tree with a large amount of viscous stationary fluid; therefore, under certain pressure
(3.0-5.0 kPa), it is time consuming to extrude the stationary fluid from the pleural surface by gas, followed by drying the stationary fluid inside the lung to produce the final inflated fixed lung specimens. Meanwhile, during the sample drying process, the large amount of viscous stationary fluid would exhibit different degrees of drying in different areas; if some sections of the lung specimen were dry, while others are not, these sections would generate pressure differences that would mildly deform the specimen (19).

The gas-liquid dual support fixation method combines inflated lung specimen fixation, formalin vapor fixation and liquid lung specimen fixation techniques. Therefore, the inflated lung fixation technique is similar to the inflated barium enema technique, in which the gas and liquid-gas are injected into the bronchial tree under certain pressure $(2.0-2.5 \mathrm{kPa})$ simultaneously (20-22). The stationary fluid coats the bronchial surface layer by layer, resulting in fixation, while the gas supports the bronchial basic framework and dries the stationary fluid on the bronchial surface. To prevent too little stationary fluid from incompletely fixing lung tissues, in the current study, the mixed stationary fluid was injected twice into the inflated lung tissues to ensure that the lung specimen was fixed sufficiently, while the internal stationary fluid was evenly distributed inside the inflated lung samples. During the drying process in this method, the substitution of liquid support by gas support was slow, ensuring that the lung stent structure would not easily contract and deform and the prepared specimen would be similar to a living specimen.

It should be noted that, in the present study, it was challenging to find lung lobes from lobectomy patients of exactly the same age, physical fitness, lesion size, lesion location, resection site, surgical procedure, and pleural damage extent, which is recommended for the Heitzman technique. The current study did not perform a detailed morphological study, therefore it must be classed as an approximating study.

The lobule structure is key to understanding HRCT images. HRCT is able to highlight various normal and abnormal characteristic changes in pulmonary lobules and has an important role in facilitating the understanding of the pathological basis, pathogenesis and diagnosis of most lung diseases, particularly interstitial lung lesions. Therefore, lobular HRCT images have been well-studied (23). As the majority of these studies were based on inflated lung specimens, there are a wide range of clinical applications for inflated lung specimens produced by the gas-liquid dual support fixation method.

Inflated lung specimens produced by the gas-liquid dual support fixation method exhibited a quality similar to the in vivo lung; therefore such specimens may be used to observe secondary pulmonary lobular structures, pulmonary lobular arteries, lobular bronchi and the interlobular septum. For example, Webb (1) used the Heitzman fixation method and proposed that the thickness of the subpleural interlobular septum was consistently $0.10-0.15 \mathrm{~mm}$. In HRCT images, the interlobular septum was very thin, exhibited a light linear shadow, had uniform thickness, and directly reached the pleural surface, and was $1.0-2.5 \mathrm{~cm}$ in length (24). However, following the comparative study of HRCT images, cord photographic images, cross-sectional gross specimens, and histological sections, it was determined that the thickness of the subpleural interlobular septum was not exactly uniform, 
although the same subpleural secondary pulmonary lobule exhibited inconsistent thickness. The interlobular septum was generally thicker at the point of conjunction with the pleura, thinner when extending towards the intra-lung, was thicker when surrounded the lobular vein, and thinner at both ends. In the same secondary pulmonary lobule, the interlobular septum was very thin in certain areas (thickness $<0.1 \mathrm{~mm}$ ) and intersected with the scan level at certain angles; therefore, these fine structures could not be differentiated by HRCT. Even on the cross-sectional gross specimen, the interlobular septum in these areas could not be distinguished by the naked eye. In certain HRCT images of the inflated fixed lung specimens, the lobular structure is visible surrounded by the complete interlobular septum, primarily due to the fact that the edema fluid caused thickening of the interlobular septum; thus, the uneven thickness of the interlobular septum appeared to be uniform. The authors of the current study plan to perform further studies investigating the lung lobular structure and pleural thickness. Inflated lung specimens prepared by the method described in the current study were used to analyze and identify the thickening of the interlobular septum, lobular core abnormalities, intralobular interstitial thickening and other abnormal shadows associated with the lung lobule, such as honeycomb (25). Through these HRCT images, it was possible to greatly improve the diagnosis and identification ranges of many lung diseases, particularly of pulmonary interstitial diseases (26).

In conclusion, by preparing inflated lung specimens using this method, the wastage of considerable amounts of clinical research materials could be avoided, thus enabling the study of subtle anatomical structures, such as the secondary pulmonary lobule, and the in-depth study of various lung diseases by using more accurate HRCT and pathological techniques. Furthermore, specimens prepared using this method enabled an in-depth study of various lung diseases by employing comparative studies of image pathology to be conducted (27). This method is not only useful for comparative studies of plain film, CT and pathological slices, but is also suitable for comparative studies of HRCT and pathology of lung-tumor interfaces and hilar area inflammation, which would increase current understanding of various lung diseases and enable the identification of the pathology of these diseases using HRCT.

\section{References}

1. Webb WR, Muller NL and Naidich DP: High-Resolution CT of the Lung. 4th edition. Lippincott Williams \& Wilkins, 2008.

2. Bamefleh $\mathrm{H}$ : Lung cancer specimen guidelines for handling and reporting. Ann Thorac Med 3: 68-73, 2008.

3. Warth A, Muley T, Meister M and Weichert W: Preanalytics in lung cancer. Recent Results Cancer Res 199: 71-84, 2015.

4. Heřmanová Z, Ctvrtlík F and Heřman M: Surface anatomy of the pulmonary fissures determined by high-resolution computed tomography. Clin Anat 25: 835-843, 2012.

5. Osarogiagbon RU, Ramirez RA, Wang CG, Miller LE, McHugh L, Adair CA, Smeltzer MP, Yu X and Berry A: Size and histologic characteristics of lymph node material retrieved from tissue discarded after routine pathologic examination of lung cancer resection specimens. Ann Diagn Pathol 18: 136-139, 2014.

6. Aumann K, Kayser G, Amann D, Bronsert P, Hauschke D, Palade E, Passlick B and Werner M: The format type has impact on the quality of pathology reports of oncological lung resection specimens. Lung Cancer 81: 382-387, 2013.
7. Hsiao SH, Chung CL, Lee CM, Chen WY, Chou YT, Wu ZH, Chen YC and Lin SE: Suitability of computed tomography-guided biopsy specimens for subtyping and genotyping of non-small-cell lung cancer. Clin Lung Cancer 14: 719-725, 2013.

8. Isaka T, Yokose T, Ito H, Imamura N, Watanabe M, Imai K, Nishii T, Woo T, Yamada K, Nakayama H and Masuda M: Comparison between CT tumor size and pathological tumor size in frozen section examinations of lung adenocarcinoma. Lung Cancer 85: 40-46, 2014.

9. Lampen-Sachar K, Zhao B, Zheng J, Moskowitz CS, Schwartz LH, Zakowski MF, Rizvi NA, Kris MG and Ginsberg MS: Correlation between tumor measurement on Computed Tomography and resected specimen size in lung adenocarcinomas. Lung Cancer 75: 332-335, 2012

10. Vanderlaan PA, Yamaguchi N, Folch E, Boucher DH, Kent MS Gangadharan SP, Majid A, Goldstein MA, Huberman MS, Kocher ON and Costa DB: Success and failure rates of tumor genotyping techniques in routine pathological samples with non-small-cell lung cancer. Lung Cancer 84: 39-44, 2014.

11. Bar J, Damianovich M, Hout Siloni G, Dar E, Cohen Y, Perelman M, Ben Nun A, Simansky D, Yellin A, Urban D and Onn A: Genetic mutation screen in early non-small-cell lung cancer (NSCLC) specimens. Clin Lung Cancer 15: 159-165, 2014.

12. Al-Zubaidi $\mathrm{N}$ and Soubani AO: Advances in diagnostic interventional pulmonology. Avicenna J Med 5: 57-66, 2015.

13. Miyata N, Endo M, Nakajima T, Kojima H, Maniwa T, Takahashi S, Isaka M, Kameya T and Ohde Y: High-resolution computed tomography findings of early mucinous adenocarcinomas and their pathologic characteristics in 22 surgically resected cases. Eur J Radiol 84: 993-997, 2015.

14. Hsia CC, Wu EY, Wagner E and Weibel ER: Preventing mediastinal shift after pneumonectomy impairs regenerative alveolar tissue growth. Am J Physiol Lung Cell Mol Physiol 281: L1279-L1287, 2001.

15. Jang IG and Kim IY: Finite Elements in Analysis and Design. Vol. 46. Elsevier, pp311-319, 2010.

16. Katashi Satoh, Takuya Kobayashi, Motoomi Ohkawa and Masatada Tanabe: Preparation of human whole lungs inflated and fixed for radiologic-pathologic correlation. Academic Radiology 4: 374-379, 1997.

17. Heitzman ER: The Lung: Radiologic-Pathologic Correlations. 2nd edition. Mosby, St. Louis, 1984.

18. Heitzman ER: Subsegmental anatomy of the lung. In: The Lung: Radiologic-Pathologic Correlations. 2nd edition. Mosby, St. Louis, pp42-49, 1984.

19. Lester SC: Manual of Surgical Pathology. 2nd edition. Elsevier, Churchill Livingstone, 2006.

20. Jagirdar J: Application of immunohistochemistry to the diagnosis of primary and metastatic carcinoma to the lung. Arch Pathol Lab Med 132: 384-396, 2008.

21. Boldrini L, Gisfredi S, Ursino S, Camacci T, Baldini E, Melfi F and Fontanini G: Mutational analysis in cytological specimens of advanced lung adenocarcinoma: A sensitive method for molecular diagnosis. J Thorac Oncol 2: 1086-1090, 2007.

22. Parwani AV, Baisden BL, Erozan YS, Burger PC and Ali SZ: Pineal gland lesions: A cytopathologic study of 20 specimens. Cancer 105: 80-86, 2005.

23. Aziz A, Ashizawa K, Nagaoki K and Hayashi K: High resolution CT anatomy of the pulmonary fissures. J Thorac Imaging 19: 186-191, 2004.

24. Andrews T and Wallace WA: Pathology of lung and pleural tumours. Surgery (Oxford) 32: 228-235, 2014.

25. Koh Y, Kenmotsu H, Serizawa M, Isaka M, Mori K, Takahashi T, Endo M, Nakajima T, Ohde Y and Yamamoto N: MC13-0079 Prospective mutational characterization of Japanese patients with non-small cell lung cancer using surgically resected tumor specimens by next-generation sequencing. Eur J Cancer 49: S12, 2013.

26. Teo B, Awan M, Coombes L and Lles S: 37 Does T staging on PET/CT correlate with pathological size of specimen in resected lung cancer? Lung Cancer 75: S12, 2012.

27. Da Cunha Santos G, Lai SW, Saieg MA, Geddie WR, Pintilie M, Tsao MS, Boerner SL and Hwang D: Cyto-histologic agreement in pathologic subtyping of non small cell lung carcinoma: Review of 602 fine needle aspirates with follow-up surgical specimens over a nine year period and analysis of factors underlying failure to subtype. Lung Cancer 77: 501-506, 2012. 